\title{
A Dedicated Orthopaedic Trauma Admission Document Significantly Improves Recording of Patient Information
}

\author{
Jefferies $\mathrm{JG}^{1^{*}}$, Brown $C D^{1}$ and Shields $D W^{2}$ \\ ${ }^{1}$ Department of Trauma and Orthopaedics, Glasgow Royal Infirmary, Glasgow, 84 Castle street, G4 OET, UK \\ ${ }^{2}$ The University of Glasgow, University Avenue, Glasgow, G12 8QQ, UK
}

*Corresponding author: Mr. James Jefferies, Department of Trauma and Orthopaedics, Glasgow Royal Infirmary, Glasgow 84 Castle Street, G4 OET, UK

\begin{abstract}
Objectives: Meticulous documentation in clinical records has been shown to improve patient care and clinician performance. Conversely, poor documentation is associated with an increased risk of adverse events. The Royal College of Surgeons (RCS) issues guidance on the contents of surgical admissions documentation.

Audit highlighted frequent omission of key criteria set out by RCS with freehand documentation. We set out to design, implement, and evaluate an orthopaedic admission proforma for our trauma patient population.

Methods: Retrospective analysis was performed of freehand clerk-ins against RCS standards. A new orthopaedic admission document was designed, and after an introductory period further retrospective analysis was performed of document clerk-ins with statistical analysis performed.

Results: 80 freehand records and 64 Admission documents were analysed. Use of proforma increased documentation in 29 out of 32 criteria set by RCS. This was statistically significant in 22 out of 29 criteria.

Conclusion: Documentation of patient information is significantly improved with use of an admission document. Clerkin quality is more consistent and comprehensive and is a quick and accessible source of information for all members of the multidisciplinary team. We have designed an effective admission document that is reproducible and an auditable tool that would benefit a range of surgical specialties and unit sizes.
\end{abstract}

\section{Keywords}

Surgical admissions, Documentation, Proforma, Orthopaedics, Trauma

\section{Introduction}

Meticulous documentation of patient information cannot be overstated and is a cornerstone of good clinical practice. Poor quality documentation is associated with a higher rate of adverse events [1], and clear and accurate documentation is linked with improved patient care and clinician performance [2].

Clear and comprehensive documentation must thus start from the moment a patient is admitted to hospital. There is good evidence to suggest that printed admissions documents aid this and have been shown to be preferred in surgery $[3,4]$ and medicine alike [5-8] over unprompted 'freehand' documentation. They have been shown to improve data retrieval time and with pointed headings act as an aide-memoire, which is of benefit to any medical or nursing professional given the time pressures and large number of tasks that contribute to a typical shift $[6,9]$.

If we consider medicolegal ramifications, more complete recording of patient information is more likely to prevent adverse events [10]. In a typically busy surgical environment where time is scarce and patient management is often decided in short time frames, comprehensive and accessible patient documentation can prevent a patient coming to harm and avoid significant consequences facing both the responsible medical individual and service.

The Royal College of Surgeons of England (RCS) issues guidance on admission documentation and what it should contain [11]. It advises inclusion of 32 key cri-

Citation: Jefferies JG, Brown CD, Shields D (2019) A Dedicated Orthopaedic Trauma Admission Document Significantly Improves Recording of Patient Information. Int Arch Orthop Surg 2:011. doi. org/10.23937/2643-4016/1710011

Accepted: June 22, 2019; Published: June 24, 2019

Copyright: (c) 2019 Jefferies JG, et al. This is an open-access article distributed under the terms of the Creative Commons Attribution License, which permits unrestricted use, distribution, and reproduction in any medium, provided the original author and source are credited. 
Table 1: Royal College of Surgeons Criteria for Documentation.

\begin{tabular}{|c|c|c|c|}
\hline Presenting Complaint & Activities of Daily Living A & Oxygen Saturations & Weight \\
\hline History of Presenting Complaint & Alcohol B & Respiratory Rate & Blood Test Results \\
\hline Past Medical History & Smoking ${ }^{B}$ & Cardiovascular Examination & Urinary Pregnancy Test ${ }^{D}$ \\
\hline Past Surgical History & Employment ${ }^{\mathrm{C}}$ & Respiratory Examination & Plan \\
\hline Medication History & Systems Review & Abdominal Examination & Advice to patient \\
\hline Allergies & Blood Pressure & Neurological Examination & Name \\
\hline Family History & Heart Rate & Abbreviated Mental test Score ${ }^{A}$ & Grade \\
\hline Package of Care ${ }^{A}$ & Temperature & Height & Time \\
\hline
\end{tabular}

${ }^{A}$ Assessed in over 65 years of age; ${ }^{B}$ Assessed in over 13 years of age; ${ }^{C}$ Assessed between 16 and 70 years of age; ${ }^{D}$ Assessed females 13-50 years of age.

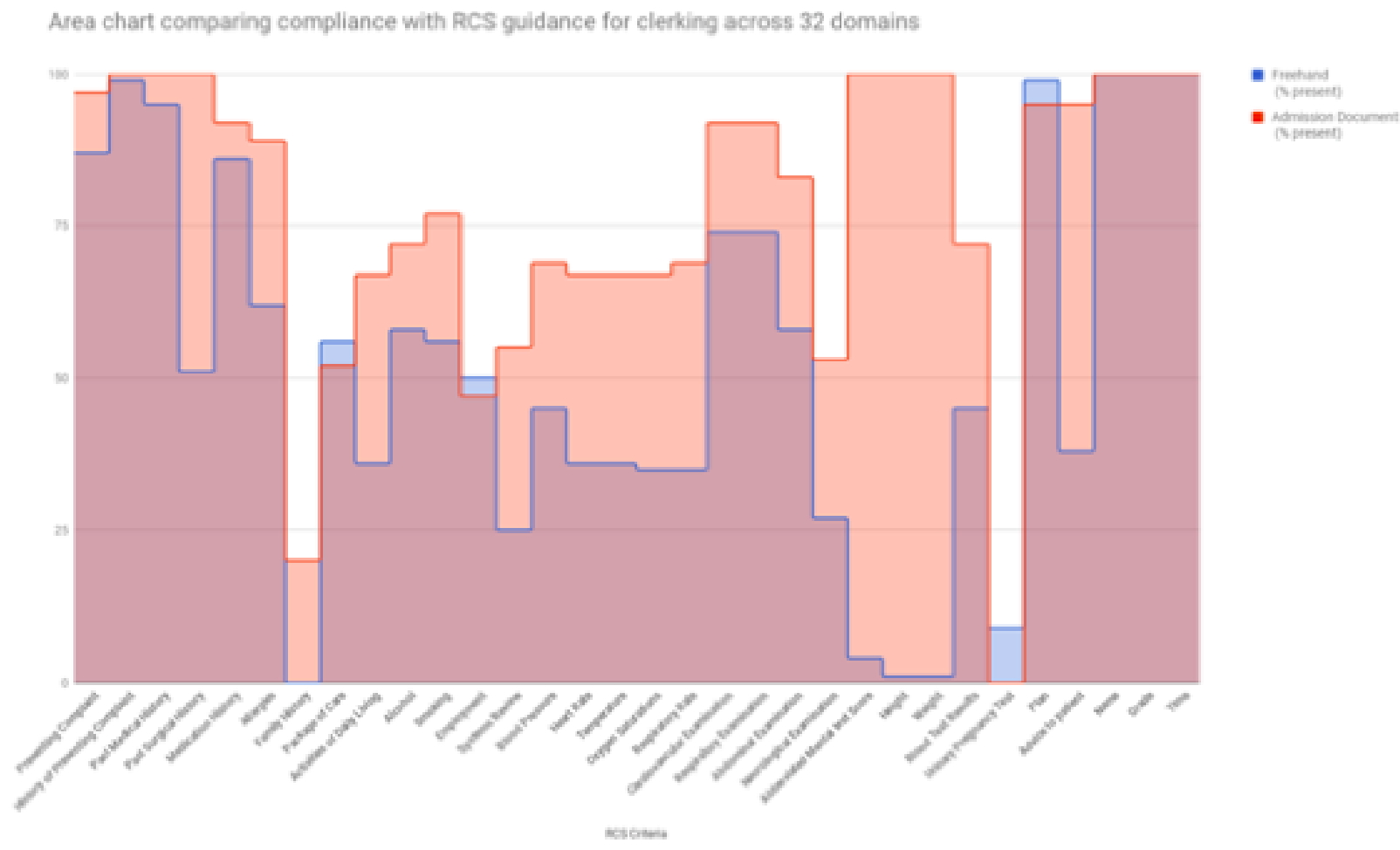

Figure 1: Area Chart comparing compliance between freehand and admission document clerk ins across 32 domains.

teria that should be documented in the admission of a surgical patient (Table 1).

The Authors work in a busy tertiary trauma unit serving a population of 1.1 million, and in one of the largest health boards in the United Kingdom [12]. Previous admission policy for patients admitted to the unit involved 'freehand' clerk ins. There was widespread variability in the completeness and quality of freehand clerk-in documentation. The authors felt that there was a need for change and desire for a document that would establish itself as the status quo for departmental admission practice.

Furthermore, literature review revealed that there is evidence to suggest the use of proformas in aiding data recording in orthopaedic operation note writing $[13,14]$. There is a paucity in the literature on comparison between freehand and admission documentation being attempted in a tertiary centre with orthopaedic patients. We therefore set out to assess whether an admission document would improve the recording of patient information according to RCS standards.

\section{Methods}

Retrospective analysis of freehand clerk-ins for trauma admissions over a two-week period against the 32 RCS criteria was performed by a single author. A new admission document was then designed and produced by the authors using RCS criteria and with further input from members of the multidisciplinary team. Final approval by departmental seniors in accordance with hospital policy was confirmed. There was uniform agreement no freehand clerk-ins would be performed for trauma patient admissions to the unit once the document was introduced Supplementary Material.

After a period of education about the document and its introduction, further retrospective analysis of clerk- 
Table 2: Results of freehand clerk-ins against the admission document.

\begin{tabular}{|c|c|c|c|c|c|c|}
\hline \multirow[t]{2}{*}{ RCS Criteria } & \multicolumn{2}{|c|}{ Freehand } & \multicolumn{2}{|c|}{ Admission Document } & \multirow[t]{2}{*}{$\%$ Change } & \multirow[t]{2}{*}{ P-Value } \\
\hline & $\mathrm{n} /$ Total & $\%$ present & n/Total & $\%$ present & & \\
\hline History of Presenting Complaint & $79 / 80$ & 99 & $64 / 64$ & 100 & +1 & 1 \\
\hline Past Medical History & $76 / 80$ & 95 & $64 / 64$ & 100 & +5 & 0.13 \\
\hline Past Surgical History & $41 / 80$ & 51 & $64 / 64$ & 100 & +96 & $<0.001$ \\
\hline Medication History & $69 / 80$ & 86 & $59 / 64$ & 92 & +7 & 0.29 \\
\hline Allergies & $50 / 80$ & 63 & $57 / 64$ & 89 & +44 & $<0.001$ \\
\hline Family History & $0 / 80$ & 0 & $13 / 64$ & 20 & +2000 & $<0.001$ \\
\hline Package of Care & $45 / 80$ & 56 & $33 / 64$ & 52 & -7 & 0.3 \\
\hline Activities of Daily Living & $29 / 80$ & 36 & $43 / 64$ & 67 & +86 & 0.83 \\
\hline Alcohol & $46 / 80$ & 58 & $46 / 64$ & 72 & +24 & $<0.001$ \\
\hline Smoking & $45 / 80$ & 56 & $49 / 64$ & 77 & +38 & $<0.001$ \\
\hline Employment & $40 / 80$ & 50 & $30 / 64$ & 47 & -6 & 0.86 \\
\hline Systems Review & $20 / 80$ & 25 & $35 / 64$ & 55 & +120 & $<0.001$ \\
\hline Blood Pressure & $36 / 80$ & 45 & $44 / 64$ & 69 & +53 & $<0.001$ \\
\hline Heart Rate & $29 / 80$ & 36 & $43 / 64$ & 67 & +86 & $<0.001$ \\
\hline Temperature & $29 / 80$ & 36 & $43 / 64$ & 67 & +86 & $<0.001$ \\
\hline Oxygen Saturations & $28 / 80$ & 35 & $43 / 64$ & 67 & +91 & $<0.001$ \\
\hline Respiratory Rate & $28 / 80$ & 35 & $44 / 64$ & 69 & +97 & $<0.001$ \\
\hline Cardiovascular Examination & $59 / 80$ & 74 & $59 / 64$ & 92 & +24 & $<0.001$ \\
\hline Respiratory Examination & $59 / 80$ & 74 & $59 / 64$ & 92 & +24 & $<0.001$ \\
\hline Abdominal Examination & $46 / 80$ & 58 & $53 / 64$ & 83 & +43 & $<0.001$ \\
\hline Neurological Examination & $22 / 80$ & 28 & $34 / 64$ & 53 & +96 & $<0.001$ \\
\hline Abbreviated Mental test Score & $3 / 80$ & 4 & $64 / 64$ & 100 & +2500 & $<0.001$ \\
\hline Height & $1 / 80$ & 1 & $64 / 64$ & 100 & +1000 & $<0.001$ \\
\hline Weight & $1 / 80$ & 1 & $64 / 64$ & 100 & +1000 & $<0.001$ \\
\hline Blood Test Results & $36 / 80$ & 45 & $46 / 64$ & 72 & +60 & $<0.001$ \\
\hline Urinary Pregnancy Test & $7 / 80$ & 9 & $0 / 64$ & 0 & -900 & 0.29 \\
\hline Plan & $79 / 80$ & 99 & $61 / 64$ & 95 & -4 & 0.33 \\
\hline Advice to patient & $30 / 80$ & 38 & $61 / 64$ & 95 & +150 & $<0.001$ \\
\hline Name & $80 / 80$ & 100 & $64 / 64$ & 100 & 0 & 1 \\
\hline Grade & $80 / 80$ & 100 & $64 / 64$ & 100 & 0 & 1 \\
\hline Time & $80 / 80$ & 100 & $64 / 64$ & 100 & 0 & 1 \\
\hline
\end{tabular}

ins using the admission document was performed over a two-week period against RCS guidelines, again by a single author. Inclusion criteria included all trauma patients admitted to the unit in that period. Exclusion criteria included clerk-ins by authors. In both evaluation cycles on-call teams completing admission documentation were unaware that they were being audited. The audit period was chosen at random and included every trauma patient admitted within the period on a consecutive, chronological basis.

Statistical analysis was performed with Fisher's Exact test using SPSS $^{\odot}$ software with a P-value of $<0.05$ deemed statistically significant.

\section{Results}

Eighty freehand clerk-ins and Sixty-Four clerk-ins using the admission document were evaluated. There was total compliance with admission document use following its introduction Table 2 and Figure 1.

In all, Documentation improved in 29 out of 32 criteria, 21 of which were statistically significant. 11 out of 13 criteria in history taking saw an improvement using the admission document, the largest increases including past surgical history (51\% against $100 \%)$, allergies (62\% v 89\%), and family history (0\% v 100\%). Of the $11 \mathrm{im}-$ provements in criteria, seven were statistically significant $(p<0.001)$ : Presenting complaint, Past Surgical History, Allergies, Family History, Alcohol intake, Smoking status, and systems review. Recording of employment and package of care status did not see an improvement with the use of the admission document.

Of aspects recorded from clinical examination, all basic observations of blood pressure, heart rate, 
temperature, oxygen saturations, and respiratory rate resulted in an improvement with the admission document, all of which were statistically significant. Recording of examinations including cardiovascular, respiratory, abdominal, and neurological systems all saw statistically significant improvements and all patients had an Abbreviated Mental Test performed in the admission document cohort compared with just $4 \%$ of freehand clerk-ins, which is of particular benefit in the orthopaedic hip fracture patient populous. Documentation of height and weight both saw improvements of $100 \%$ recording in admission document against 1\% apiece in the freehand cohort. This was significant $p<0.001$.

The admission document improved recording of blood test results $72 \%$ compared with $45 \%$ of freehand clerk ins $(p<0.001)$. The clerk in document did not show an improvement in recording pregnancy test results with $9 \%$ freehand versus $0 \%$ in the admission document, however this is a likely consequence of the infrequent use of the test.

Administrative aspects of documentation; name and grade of clerking doctor, time of clerk-in, and recording of a plan was consistently done well in both freehand and admission document clerk-ins with $100 \%$ inclusion in both. Documented advice to patients saws a significant improvement from $38 \%$ freehand to $95 \%$ with the admission document. Recording of a plan was performed in $99 \%$ freehand clerk ins compared with $95 \%$ in the admission document cohort.

\section{Discussion}

Our results have shown recording of patient information on admission to our unit is significantly superior with the application of a dedicated admission document. Its implementation has been positively received by the unit and wider ranging medical and nursing professionals. The objective results match that of previous studies $[2,3]$ that highlight admission documents can improve recording patient information in both medical and surgical patients. Whilst Faraj, et al. explored the use of an admissions proforma for elderly trauma patients, not based on RCS guidance but designed according to the departmental needs of a regional unit [4], this is the first to describe its use and success in a major trauma centre. Our results are an advance on previous studies [2,3]. Using RCS guidance, with more improvements in criteria recording and more statistically significant results in our orthopaedic admission document.

The quality of clerk-ins was more thorough and consistent. Key criteria saw an improvement in documentation as colleagues were prompted by the flowing and comprehensive design, which in support of previous studies can act as an aide memoire [6]. Furthermore, it acts as a beacon of readily available, comprehensive information for members of the MDT including, but not exhaustive to Anaesthetists, Physiotherapists and Occu- pational therapists, and retrieval of information using the document can be performed with ease and is quicker than sifting through single pieces of freehand notes.

The admission document is readily auditable and easily changed if the need arises or guidance makes for further recommendations.

A limitation our study lies in that although colloquial feedback of the new admission document was well received and positive, Questionnaires to MDT colleagues and doctors using the document would have been of benefit in subjectively supporting our findings in comparing the two forms of clerk-ins. However, it would be elementary to audit the document in the future and include a subjective questionnaire.

\section{Conclusion}

We have shown that a new admission document can be successfully implemented with profound results. The document is a focus for a wealth of vital patient information that should be included in all admissions, and acts to streamline the search for patient information that is of benefit to a variety of medical and nursing professionals during the pre, peri, and postoperative chapters of patient admissions.

We believe our work is easily reproducible, and although designed for orthopaedic patients, would be of benefit to a range of surgical specialties and in departments of varying size and pressures.

\section{Compliance with Ethical Standards}

\section{Conflict of interest}

The authors declare that they have no conflict of interest.

\section{Funding}

This research did not receive any specific grant from funding agencies in the public, commercial, or not-forprofit sector.

\section{Ethical approval}

This article does not contain any studies with human participants or animals performed by any of the authors.

\section{Supplementary Material}

Trauma Admission Document.

\section{References}

1. Zegers $M$, de Bruijne $M C$, Spreeuwenberg $P$, Wagner $C$, Groenewegen PP, et al. (2011) Quality of patient record keeping: an indicator of the quality of care? BMJ Qual Saf 20: 314-318.

2. Mann R, Williams $J(2003)$ Standards in medical record keeping. Clin Med 3: 329-332.

3. Ehsanullah J, Ahmad U, Solanki K, Healy J, Kadoglou N (2015) The Surgical Admissions proforma: Does it make it difference? Ann Med Surg (Lond) 2015: 53-57. 
4. Faraj $A A$, Brewer OD, Afinowi $R$ (2011) The value of an admissions proforma for elderly patients with trauma. Injury 42: $171-172$

5. Belmin $J$, de la Fournière $F$, Bellot $P$, Medjahed $S$, SibonyPrat J, et al. (1998) Quality of the information collected during admission to a hospital geriatric service: importance of a structured medical record. Presse Med 27: 1519-1522.

6. O'Driscoll BR, Al-Nuaimi D (2003) Medical admission records can be improved by the use of a structured proforma. Clin Med 3: 385-386.

7. Irtiza-Ali A, Houghton CM, Raghuram A, O'Driscoll BR (2001) Medical admissions can be made easier, quicker and better by the use of a pre-printed medical admission proforma. Clin Med 1: 327.

8. Diver AJ, Craig BF (2005) Admission proforma significantly improves the medical record. Scott Med J 50: 101-102.
9. Murphy BJ (2001) Principles of good medical record documentation. J Med Pract Manage 16: 258-260.

10. RJ Lilford, MA Mohammed, D Braunholtz, T P Hofer (2003) The measurement of active errors: methodological issues. Qual Saf Health Care 12: 8-12.

11. Surgeons TRCo (1994) Guidelines for clinicians on medical records and notes. RCSEng- Professional standards and regulation.

12. (2017) Who are we, what we do. NHSGGC.

13. Din R, Jenna D, Muddu BN (2001) The use of an aidememoire to improve quality of operation notes in an orthopaedic unit. Ann R Coll Surg Engl 83: 319-320.

14. McGregor-Riley J, Ali F, Al Hussainy H, Sukumar S (2003) Proformas can improve the quality of orthopaedic operation notes. J Bone Joint Surg Br 85: 124. 